TRANSACTIONS OF THE

AMERICAN MATHEMATICAL SOCIETY

Volume 300, Number 2, April 1987

\title{
THE MACRAE INVARIANT AND THE FIRST LOCAL CHERN CHARACTER
}

\author{
PAUL ROBERTS
}

\begin{abstract}
The first local Chern character of a bounded complex of locally free sheaves on a scheme $Y$ is given by intersection with a Cartier divisor. In the case of the resolution of a module of finite projective dimension, this is the invariant defined by MacRae.
\end{abstract}

Let $R$ be a commutative Noetherian ring, and let $M$ be a finitely generated module of finite projective dimension. In his investigation of invariants of these modules, MacRae [6] constructed an invertible ideal $G(M)$ associated to $M$ which describes the part of the support of $M$ of codimension 1 . The fact that $G(M)$ is invertible implies many properties of the support of $M$, and it has recently been used by Foxby [3] to prove some conjectures on intersection multiplicities of modules of finite projective dimension with modules of Krull dimension one.

In this paper we generalize the construction of MacRae to a bounded complex $E_{*}$ of locally free sheaves on a Noetherian scheme $Y$ and show that this can be used to describe the first local Chern character of $E_{*}$. A bounded complex of locally free sheaves of finite rank will be called a perfect complex. For technical reasons, we assume that $Y$ is connected and quasi-projective over an affine scheme. Let $X$ be the support of $E_{*}$, denoted $\operatorname{Supp}\left(E_{*}\right)$; this can be defined as the set of points of $Y$ where $E_{*}$ is not exact, or, equivalently, as the union of the supports of the homology modules $H_{i}\left(E_{*}\right)$. We assume that $X$ is contained in some Cartier divisor. Locally, this means that the ideal defining $X$ contains a non-zero-divisor, and if $E_{*}$ is a resolution of a module, our assumption follows whenever $X$ is a proper subset of $Y$. An equivalent formulation of this condition is that $X$ contains no points $y$ of $Y$ such that the local ring $\mathcal{O}_{y}$ has depth zero; such a point will be called a point of depth zero. In this situation we construct a Cartier divisor $G\left(E_{*}\right)$ on $Y$ generalizing the MacRae invariant.

If $Y$ is quasi-projective over a regular local ring (this includes, among others, the case where $Y=\operatorname{Spec} R$ and $R$ is a complete local ring), there is a theory of local Chern characters defined for perfect complexes on $Y$. For a complex $E_{*}$ with support $X$ as above, for any scheme $Y^{\prime}$ together with a map of finite type $f: Y^{\prime} \rightarrow Y$, and for integers $n$ and $j$, the $j$ th local Chern character $\operatorname{ch}_{j}\left(E_{*}\right)$ defines an

Received by the editors December 4, 1985 and, in revised form, March 11, 1986.

1980 Mathematics Subject Classification (1985 Revision). Primary 14C17; Secondary 13D15, 13D25, $13 \mathrm{H} 15,14 \mathrm{C} 35$.

Supported in part by a grant from the National Science Foundation. 
"intersection operator"

$$
\operatorname{ch}_{j}\left(E_{*}\right): A_{n}\left(Y^{\prime}\right) \rightarrow A_{n-j}\left(f^{-1}(X)\right),
$$

where, for any scheme $Z, A_{*}(Z)=A_{*}(Z) \otimes Q$ is the rational Chow group of cycles on $Z$ modulo rational equivalence. We refer to Fulton [4, Chapter 18] for definitions and properties of these operators. We show below that in our situation $\operatorname{ch}_{1}\left(E_{*}\right)$ is the operator defined by intersecting with the Cartier divisor $G\left(E_{*}\right)$. This implies that if the codimension of $X$ in $Y$ is at least 2, then $\operatorname{ch}_{1}\left(E_{*}\right)=0$, a result which is used in Roberts [7] to prove a vanishing conjecture on multiplicities for rings with singular locus of dimension 1. It should be remarked that in higher codimensions the corresponding statement is not true; Dutta, Hochster, and McLaughlin [2] have constructed an example in which the codimension of the support of $E_{*}$ is 3 , but $\operatorname{ch}_{2}\left(E_{*}\right) \neq 0$.

An invariant similar to the one described here has been constructed for complexes by Iversen [8]; his is an element of the local cohomology group $H_{Z}^{1}(\mu)$, where $Z$ contains the support of $E_{*}$ and $\mu$ is the sheaf of units. He also outlines a proof that this gives the first Chern class in étale cohomology; in this context, it appears to be possible to reduce the "global" case; a technique which has not been successfully carried out for the Chern characters defined as operators in the Chern group which we use here.

Before constructing $G\left(E_{*}\right)$, it is necessary to deal with one technical point. We need to know that the following two constructions are possible:

1. If $K$ is a coherent sheaf on $Y$, then there is a locally free sheaf $F$ and a (locally) surjective map: $F \rightarrow K$.

2. Suppose we are given a finite subset $T$ of $Y$ containing all points of depth zero. If we have a diagram of maps of locally free sheaves:

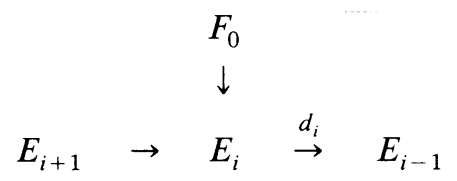

such that $\operatorname{Im}\left(F_{0}\right) \subseteq \operatorname{Ker} d_{i}, F_{0}$ has rank $r$, and the support of $H_{i}\left(E_{*}\right)$ contains no points of $T$, then there is a locally free sheaf $F_{1}$, also of rank $r$, together with maps

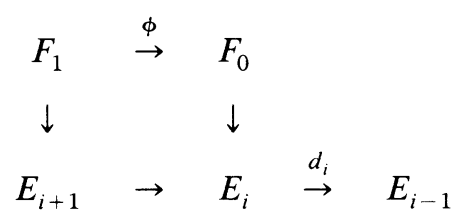

such that the diagram commutes and $\operatorname{Supp}(\operatorname{Coker} \phi)$ contains no points of $T$.

If $Y$ is affine, the first construction is accomplished by mapping generators of a free module onto generators of $K$, and the second by choosing elements of $F_{0}$ which map to the image of $d_{i+1}$ and which avoid the prime ideals corresponding to points of $T$. The first construction is in fact standard in more generality; a proof can be found in Borelli [1]. The method of proof is to find a line bundle $L$ on $Y$ and a 
global section $s$ of $L$ such that $Y_{s}=\{y \in Y \mid s$ does not generate $L$ at $y\}$ is affine. One makes the construction on $Y_{s}$ using $\mathcal{O}_{Y_{s}}^{k}$ as above, then, using EGA $I$ (9.3.1) (reference [5]), one deduces the construction on $Y$ using $\left(L^{\otimes(-m)}\right)^{k}$ for some integer $m$, at least up to the support of $s$. The same method works for the second construction, provided that $L$ and $s$ can always be chosen so that the support of $s$ contains no point of $T$; if $Y$ is quasi-projective over an affine scheme, this can always be done.

We now define $G\left(E_{*}\right)$ when $E_{*}$ is a perfect complex of length 1 . In this case $E_{*}$ is just a map between locally free sheaves of the same rank (recall that $\operatorname{Supp}\left(E_{*}\right)$ is always assumed to contain no points of depth zero). Let

$$
E_{*}=\cdots \rightarrow 0 \rightarrow E_{i+1} \stackrel{d_{i+1}}{\rightarrow} E_{i} \rightarrow 0 \rightarrow \cdots
$$

with $\operatorname{rank}\left(E_{i}\right)=\operatorname{rank}\left(E_{i+1}\right)=r$. We then have a map

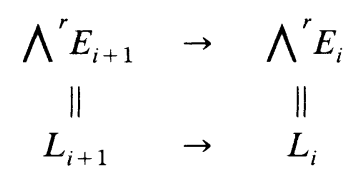

where $L_{i+1}$ and $L_{i}$ are locally free sheaves of rank one. These give a map $L_{i+1} \otimes L_{i}^{-1} \rightarrow \mathcal{O}_{Y}$.

The image of this map is a Cartier divisor $D$, with $L_{i+1} \otimes L_{i}^{-1} \cong \mathcal{O}_{Y}(-D)$, and we define $G\left(E_{*}\right)$ to be $(-1)^{i} D$ (using additive notation for divisors). Locally, the map on $r$ th exterior powers is given by the determinant of a matrix defining $d_{i+1}$, so this is the same as the MacRae invariant for a module of projective dimension 1.

Proposition 1. Let $E_{*}$ be a perfect complex of length 1 . If $\alpha$ is a cycle in $A_{n} Y^{\prime}$, where $f: Y^{\prime} \rightarrow Y$ is a map of schemes, then

$$
\operatorname{ch}_{1}\left(E_{*}\right)(\alpha)=G\left(E_{*}\right) \cap \alpha \quad \text { in } A_{n-1}\left(f^{-1}(X)\right) .
$$

Proof. Since all of the operations used in defining $G\left(E_{*}\right)$ are compatible with pullbacks, we can assume that $Y^{\prime}=Y$, and, replacing $Y^{\prime}$ by a component of $\alpha$, we can assume that $Y$ is an integral scheme of dimension $n$ and $\alpha=[Y]$. The proof is divided into two cases.

Case 1. $X=Y$. In this case,

$$
\operatorname{ch}_{1}\left(E_{*}\right)=(-1)^{i}\left(\operatorname{ch}_{1}\left(E_{i}\right)-\operatorname{ch}_{1}\left(E_{i+1}\right)\right) .
$$

We use the equality

$$
\operatorname{ch}_{1}\left(E_{j}\right)=c_{1}\left(E_{j}\right)=c_{1}\left(\bigwedge^{r} E_{j}\right)=\operatorname{ch}_{1}\left(\bigwedge^{r} E_{j}\right),
$$

where $c_{1}(E)$ denotes the first Chern class of $E$ (see Fulton [4, Remark 3.2.3]). Thus

$$
\begin{aligned}
\operatorname{ch}_{1}\left(E_{*}\right)(\alpha) & =(-1)^{i}\left(c_{1}\left(L_{i}\right)-c_{1}\left(L_{i+1}\right)\right)(\alpha)=(-1)^{i} c_{1}\left(L_{i} \otimes L_{i+1}^{-1}\right)(\alpha) \\
& =(-1)^{i} c_{1}(\mathcal{O}(D))(\alpha)=G\left(E_{*}\right) \cap \alpha .
\end{aligned}
$$


Case 2. $X \neq Y$. In this case $X$ is a proper subset of $Y$, and, since $\operatorname{ch}_{1}\left(E_{*}\right)[Y]$ is a cycle of codimension 1, we can localize and assume that $Y=\operatorname{Spec} R$, where $R$ is a local domain of dimension 1 and $X$ is the closed point of $Y$. By normalizing, we can then assume that $R$ is a discrete valuation ring; let $t$ generate its maximal ideal. Then the complex $E_{*}$ decomposes into a sum of complexes $\cdots 0 \rightarrow R \stackrel{t^{m}}{\rightarrow} R \rightarrow 0$, and it suffices to prove the result in this case. However, in this case both sides can easily be computed and we obtain

$$
\operatorname{ch}_{1}\left(E_{*}\right)([Y])=m[X]=G\left(E_{*}\right) \cap[Y] .
$$

Proposition 2. Let $0 \rightarrow E_{*}^{\prime} \rightarrow E_{*} \rightarrow E_{*}^{\prime \prime} \rightarrow 0$ be a short exact sequence of perfect complexes of length 1 with $E_{j}^{\prime}=E_{j}=E_{j}^{\prime \prime}=0$ for $j \neq i, i+1$. Then $G\left(E_{*}\right)=G\left(E_{*}^{\prime}\right)$ $+G\left(E_{*}^{\prime \prime}\right)$.

Proof. Let $r^{\prime}, r$, and $r^{\prime \prime}$ be the ranks of $E_{i}^{\prime}, E_{i}$, and $E_{i}^{\prime \prime}$ respectively. We then have $r=r^{\prime}+r^{\prime \prime}$, and

$$
\bigwedge^{r} E_{j} \cong \wedge^{r^{\prime}} E_{j}^{\prime} \otimes \bigwedge^{r^{\prime \prime}} E_{j}^{\prime \prime}
$$

or

$$
L_{j} \cong L_{j}^{\prime} \otimes L_{j}^{\prime \prime} \quad \text { for } j=i, i+1 .
$$

Furthermore, the embedding $L_{i+1} \rightarrow L_{i}$ factors as follows:

$$
L_{i+1} \cong L_{i+1}^{\prime} \otimes L_{i+1}^{\prime \prime} \rightarrow L_{i+1}^{\prime} \otimes L_{i}^{\prime \prime} \rightarrow L_{i}^{\prime} \otimes L_{i}^{\prime \prime} \cong L_{i} .
$$

The corresponding Cartier divisors of these factors are $G\left(E_{*}^{\prime \prime}\right)$ and $G\left(E_{*}^{\prime}\right)$ respectively, so the assertion follows.

Now that $G\left(E_{*}\right)$ has been defined for complexes of length 1, the general case can be defined by approximating a general complex by complexes of length 1 . More precisely, let $E_{*}=0 \rightarrow E_{k} \rightarrow \cdots \rightarrow E_{m} \rightarrow 0$, so that $k$ is the largest integer $j$ for which $E_{j} \neq 0$, and let $i$ be the smallest integer $j$ for which $H_{j}\left(E_{*}\right) \neq 0$. We define $G\left(E_{*}\right)$ by induction on $k-i$.

It is impossible for $k-i$ to equal zero, since the support of $E_{*}$ contains no points of depth zero.

If $k-i=1$, we map a free module $F_{k-1}$ to the kernel of $d_{k-1}$ so that the map from $F_{k-1}$ to $H_{k-1}\left(E_{*}\right)$ is surjective. We then have a diagram

$$
\begin{gathered}
F_{k-1} \\
\downarrow \phi \\
\\
\quad \begin{array}{ll}
\downarrow \\
\end{array} \stackrel{d_{k}}{\rightarrow} \quad E_{k-1} \quad \rightarrow E_{k-2} \rightarrow \cdots .
\end{gathered}
$$

Let $F_{k}=\phi^{-1}\left(\operatorname{Im}\left(d_{k}\right)\right)$. Since $H_{k-1}\left(E_{*}\right)$ has a resolution by locally free sheaves which stops in degree $k$, we deduce that $F_{*}$ is locally free, and we define $G\left(E_{*}\right)=$ $G\left(F_{*}\right)$, where $F_{*}$ is the complex $0 \rightarrow F_{k} \rightarrow F_{k-1} \rightarrow 0$.

If $k-i>1$, we map a locally free sheaf $F_{i}$ to $\operatorname{Ker} d_{i}$ so that it induces a surjection onto $H_{i}\left(E_{*}\right)$ as above. If the rank of $F_{i}$ is $r$, we can find another locally 
free sheaf $F_{i+1}$ of rank $r$ so that we have a diagram

$$
\begin{array}{rccc}
0 \rightarrow & F_{i+1} & \stackrel{f}{\rightarrow} & F_{i} \\
& \downarrow \phi_{i+1} & & \downarrow \phi_{i} \\
\cdots \rightarrow E_{i+2} \rightarrow & E_{i+1} & \rightarrow & E_{i} \rightarrow E_{i-1} \rightarrow \cdots .
\end{array}
$$

In addition, if $T$ is any finite subset of $Y$ disjoint from $X$ and containing all points of depth zero, we can assume that $\operatorname{Supp}(\operatorname{Coker} f) \cap T$ is empty. Let $C_{*}$ be the mapping cone of $\phi: F_{*} \rightarrow E_{*}$. From the long exact sequence

$$
\cdots \rightarrow H_{j}\left(F_{*}\right) \rightarrow H_{j}\left(E_{*}\right) \rightarrow H_{j}\left(C_{*}\right) \rightarrow H_{j-1}\left(F_{*}\right) \rightarrow \cdots
$$

and the surjectivity of $H_{i}\left(F_{*}\right) \rightarrow H_{i}\left(E_{*}\right)$ we deduce that $H_{j}\left(C_{*}\right)=0$ for $j \leqslant i$. Furthermore, since $i-k \geqslant 2, C_{j}=0$ for $j>k$. Hence, by induction, we have defined $G\left(C_{*}\right)$. Let $G\left(E_{*}\right)=G\left(C_{*}\right)+G\left(F_{*}\right)$.

There are a number of choices in this definition, but the next theorem shows that the result is independent of these choices.

THEOREM 1. (a) $G\left(E_{*}\right)$ is independent of the choices of complexes $F_{i+1} \rightarrow F_{i}$ and maps into $E_{*}$ used in the construction.

(b) If $0 \rightarrow E_{*}^{\prime} \rightarrow E_{*} \rightarrow E_{*}^{\prime \prime} \rightarrow 0$ is a short exact sequence of perfect complexes, then $G\left(E_{*}\right)=G\left(E_{*}^{\prime}\right)+G\left(E_{*}^{\prime \prime}\right)$.

Proof. We prove both of these assertions by induction on $k-i$, where $k$ and $i$ are defined as above for (a), while for (b) we let $k=\sup \left\{j \mid E_{j}, E_{j}^{\prime}\right.$, or $\left.E_{j}^{\prime \prime} \neq 0\right\}$ and $i=\inf \left\{j \mid H_{j}\left(E_{*}\right), H_{j}\left(E_{*}^{\prime}\right)\right.$, or $\left.H_{j}\left(E_{*}^{\prime \prime}\right) \neq 0\right\}$.

As before, the lowest value of $k-i$ which must be considered is $k-i=1$.

To prove (a) in this case, suppose that $F_{k-1}$ and $G_{k-1}$ are surjective maps of locally free sheaves into $\operatorname{Ker}\left(d_{k-1}\right)$ which induce surjections onto $H_{k-1}\left(E_{*}\right)$. Replacing $F_{k-1}$ with $F_{k-1} \oplus G_{k-1}$, we can assume that there is a map $f_{k-1}$ from $F_{k-1}$ onto $G_{k-1}$. The map $f_{k-1}$ will induce a map $f_{*}$ on kernels, and we have

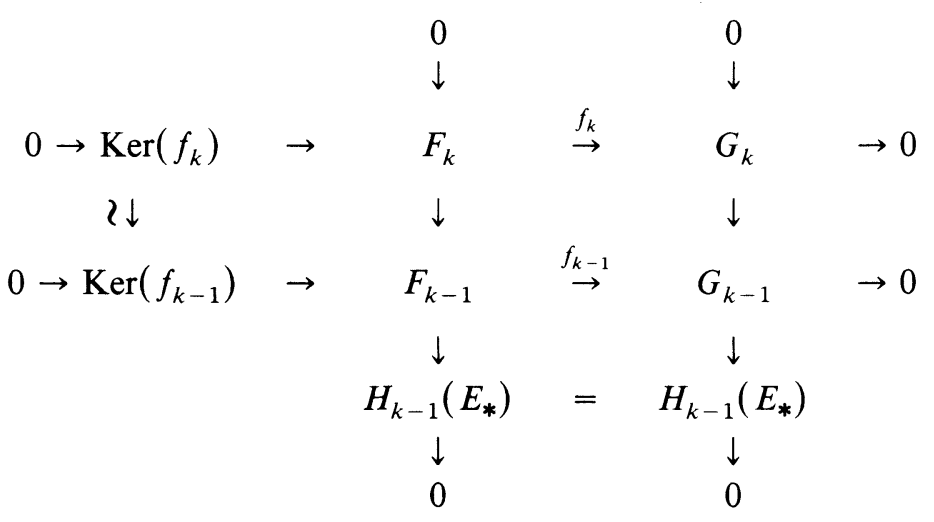

with exact rows and columns. Statement (a) now follows from Proposition 2 applied to the short exact sequence of complexes in the top two rows. 
To prove (b) in this case, we note that we have a short exact sequence

$$
0 \rightarrow H_{k-1}\left(E_{*}^{\prime}\right) \rightarrow H_{k-1}\left(E_{*}\right) \rightarrow H_{k-1}\left(E_{*}^{\prime \prime}\right) \rightarrow 0 .
$$

Let $F_{k-1}^{\prime}$ be a locally free sheaf mapping onto $H_{k-1}\left(E_{*}^{\prime}\right)$, and let $F_{k-1}^{\prime \prime}$ map onto $H_{k-1}\left(E_{*}\right)$. We form the diagram

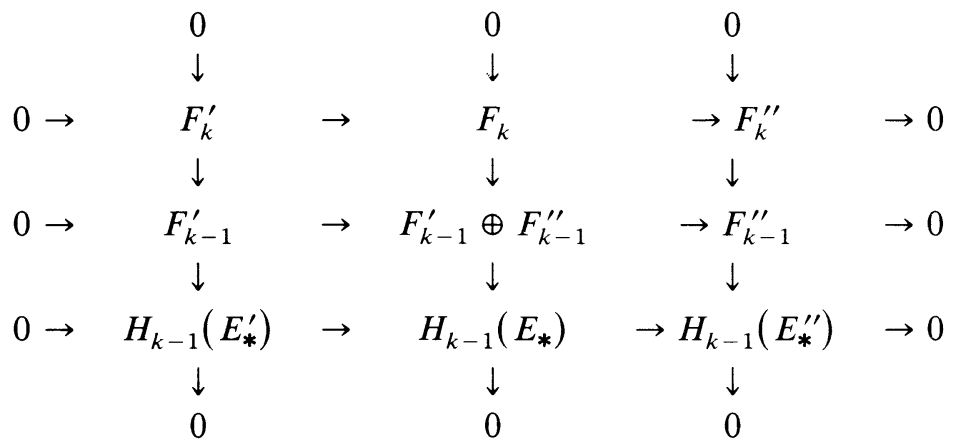

where the top row consists of the kernels of the vertical maps. From (a), we can use these complexes to compute $G\left(E_{*}^{\prime}\right), G\left(E_{*}\right)$, and $G\left(E_{*}^{\prime \prime}\right)$, and (b) now follows from Proposition 2.

We now prove (a) by induction, assuming that $k-i=s \geqslant 2$ and that (a) and (b) hold whenever $k-i<s$. Let $F_{*} \rightarrow E_{*}$ and $G_{*} \rightarrow E_{*}$ be two maps of complexes of length 1 to $E_{*}$ as in the above construction. We then have a map $F_{*} \oplus G_{*} \rightarrow E_{*}$ of the same type, and it suffices to compare the definition of $G\left(E_{*}\right)$ by means of $F_{*} \oplus G_{*}$ with that by means of $G_{*}$. Denote $C_{*}^{F}$ the mapping cone of $F_{*} \rightarrow E_{*}$ and $C_{*}^{F \oplus G}$ the mapping cone of $F_{*} \oplus G_{*} \rightarrow E_{*}$. We have exact sequences

$$
0 \rightarrow F_{*} \rightarrow F_{*} \oplus G_{*} \rightarrow G_{*} \rightarrow 0
$$

and

$$
0 \rightarrow C_{*}^{G} \rightarrow C_{*}^{F \oplus G} \rightarrow F_{*}[-1] \rightarrow 0 .
$$

We thus have

$$
\begin{aligned}
&\left.G\left(E_{*}\right) \text { (computed from } G_{*}\right)=G\left(C_{*}^{G}\right)+G\left(G_{*}\right) \\
&=\left(G\left(C_{*}^{F \oplus G}\right)-G\left(F_{*}[-1]\right)\right)+\left(G\left(F_{*} \oplus G_{*}\right)-G\left(F_{*}\right)\right) \\
& \quad \quad \text { by induction and Proposition 2) } \\
&=G\left(C_{*}^{F \oplus G}\right)+G\left(F_{*} \oplus G_{*}\right) .
\end{aligned}
$$

This completes the proof of (a).

We now prove (b), assuming that (a) holds for $k-i \leqslant s$ and that (b) holds for $k-i<s$. Take complexes $F_{*}^{\prime} \rightarrow E_{*}^{\prime}$ and $F_{*}^{\prime \prime} \rightarrow E_{*}$ as in the construction of $G\left(E_{*}^{\prime}\right)$ and $G\left(E_{*}\right)$. Since $H_{i-1}\left(E_{*}^{\prime}\right)=0$, the map from $H_{i}\left(E_{*}\right)$ to $H_{i}\left(E_{*}^{\prime \prime}\right)$ is surjective, and the composition $F_{*}^{\prime \prime} \rightarrow E_{*} \rightarrow E_{*}^{\prime \prime}$ can be used to define $G\left(E_{*}^{\prime \prime}\right)$ (this uses assertion (a) for $k-i=s$ ). We now take the diagram of maps of complexes

$$
\begin{array}{ccccccc}
0 \rightarrow & F_{*}^{\prime} & \rightarrow & F_{*}^{\prime} \oplus F_{*}^{\prime \prime} & \rightarrow & F_{*}^{\prime \prime} & \rightarrow 0 \\
& \downarrow & & \downarrow & & \downarrow & \\
0 \rightarrow & E_{*}^{\prime} & \rightarrow & E_{*} & \rightarrow & E_{*}^{\prime \prime} & \rightarrow 0 .
\end{array}
$$


If $C_{*}^{\prime}, C_{*}$, and $C_{*}^{\prime \prime}$ are the respective mapping cones, there is a short exact sequence $0 \rightarrow C_{*}^{\prime} \rightarrow C_{*} \rightarrow C_{*}^{\prime \prime} \rightarrow 0$. The result now follows from the induction hypothesis applied to this sequence and from Proposition 2.

The uniqueness of $G\left(E_{*}\right)$ is needed in the next result.

THEOREM 2. Let $E_{*}$ be a perfect complex on $Y$ with support $X$. If $f: Y^{\prime} \rightarrow Y$ is any map of finite type, and if $\alpha \in A_{n}\left(Y^{\prime}\right)$, then $\operatorname{ch}_{1}\left(E_{*}\right)(\alpha)=G\left(E_{*}\right) \cap \alpha$ in $A_{n-1}\left(f^{-1}(X)\right)$.

Proof. As before, we can assume that $Y^{\prime}$ is an integral scheme of dimension $n$ and that $\alpha=\left[Y^{\prime}\right]$. The proof is by induction on $k-i$, where, as above, $k=$ $\sup \left\{j \mid E_{j} \neq 0\right\}$ and $i=\inf \left\{j \mid H_{j}\left(E_{*}\right) \neq 0\right\}$. If $k-i=1$, then the construction of $G\left(E_{*}\right)$ gives a quasi-isomorphism $F_{*} \rightarrow E_{*}$. Thus

$$
\begin{aligned}
\operatorname{ch}_{1}\left(E_{*}\right)(\alpha) & =\operatorname{ch}_{1}\left(F_{*}\right)(\alpha) \\
& =G\left(F_{*}\right) \cap \alpha \quad \text { by Proposition 1 } \\
& =G\left(E_{*}\right) \cap \alpha \quad \text { by definition. }
\end{aligned}
$$

Assume now that $k-i>1$.

Case 1. The function $f$ maps $Y^{\prime}$ into $X$. Let $F_{*} \rightarrow E_{*}$ be as in the inductive construction of $G\left(E_{*}\right)$. The short exact sequence

$$
0 \rightarrow f^{*}\left(E_{*}\right) \rightarrow f^{*}\left(C_{*}\right) \rightarrow f^{*}\left(F_{*}[-1]\right) \rightarrow 0
$$

gives the equation

$$
\operatorname{ch}_{1}\left(E_{*}\right)\left[Y^{\prime}\right]=\operatorname{ch}_{1}\left(C_{*}\right)\left[Y^{\prime}\right]+\operatorname{ch}_{1}\left(F_{*}\right)\left[Y^{\prime}\right]
$$

in $A_{n-1}\left(Y^{\prime}\right)$. By definition, we have $G\left(E_{*}\right)=G\left(C_{*}\right)+G\left(F_{*}\right)$, so that

$$
G\left(E_{*}\right) \cap\left[Y^{\prime}\right]=G\left(C_{*}\right) \cap\left[Y^{\prime}\right]+G\left(F_{*}\right) \cap\left[Y^{\prime}\right]
$$

in $A_{n-1}\left(Y^{\prime}\right)$. By induction, the result is true for $C_{*}$ and $F_{*}$, so it is true for $E_{*}$. Since $f$ maps $Y^{\prime}$ into $X, Y^{\prime}=f^{-1}(X)$, and this proves the result.

Case 2. Suppose now that $f\left(Y^{\prime}\right) \nsubseteq X$. Let $y$ be the generic point of $\overline{f\left(Y^{\prime}\right)}$ in the scheme $Y$. Since $y \notin X$, in the inductive construction of $G\left(E_{*}\right)$, we can assume that $y \notin \operatorname{Supp}\left(F_{*}\right)$. Let $\bar{X}=\operatorname{Supp}\left(F_{*}\right) \cup X$. Then, using the short exact sequence

$$
0 \rightarrow E_{*} \rightarrow C_{*} \rightarrow F_{*}[-1] \rightarrow 0,
$$

we have that

$$
\operatorname{ch}_{1}\left(E_{*}\right)\left[Y^{\prime}\right]=\operatorname{ch}_{1}\left(C_{*}\right)\left[Y^{\prime}\right]+\operatorname{ch}_{1}\left(F_{*}\right)\left[Y^{\prime}\right]
$$

in $A_{n-1}\left(f^{-1}(\bar{X})\right)$. Since $y \notin \operatorname{Supp}\left(F_{*}\right), f^{-1}(\bar{X})$ is a proper closed subset of $Y^{\prime}$, and any integral subscheme of $f^{-1}(\bar{X})$ of dimension $n-1$ is a component. Hence the coefficients of each component in $\operatorname{ch}_{1}\left(E_{*}\right)\left[Y^{\prime}\right]$ and $G\left(E_{*}\right) \cap\left[Y^{\prime}\right]$ must be equal, and, since we could exclude any component not lying in $f^{-1}(X)$ in the same way as we excluded $Y^{\prime}$, the coefficients of these components must be zero. Hence

$$
\operatorname{ch}_{1}\left(E_{*}\right)\left[Y^{\prime}\right]=G\left(E_{*}\right) \cap\left[Y^{\prime}\right] \text { in } A_{n-1}\left(f^{-1}(X)\right)
$$

as required.

We now use Theorem 2 to show that $\operatorname{ch}_{1}\left(E_{*}\right)$ vanishes if the codimension of $X$ is greater than 1 . However, we first show that $G\left(E_{*}\right)$ may itself not vanish in this case. 
EXAMPLE. Let $Y=\operatorname{Spec} R$, where $R=K[[X, Y, S, T]] /(X, Y) \cap(S, T)$. Let $E_{*}$ be the complex

$$
R \stackrel{\left(\bar{X}^{Y+S}\right)}{\rightarrow} R^{2} \stackrel{(X+\alpha S, Y+\alpha T)}{\rightarrow} R
$$

where $\alpha \neq 0,1$ is an element of $K$. Then $G\left(E_{*}\right)$ is defined by $(X+\alpha S) /(X+S)$, which is not a unit in $R$. However, the support of $E_{*}$ has codimension two. It can be verified directly that $\operatorname{ch}_{1}\left(E_{*}\right)=0$ in this case; it also follows from the third case of the following theorem.

THEOREM 3. Let $E_{*}$ be a perfect complex on $Y$ with support $X$. Assume that one of the following three conditions holds:

(a) $Y=\operatorname{Spec} R$, where $R$ is a local ring, $E_{*}$ is the resolution of an $R$-module $M$, and $\operatorname{dim} R-\operatorname{dim} M \geqslant 2$.

(b) $Y$ is normal, and $\operatorname{dim} Y-\operatorname{dim} X \geqslant 2$.

(c) For every component $Z$ of $Y, \operatorname{dim} Z-\operatorname{dim}(X \cap Z) \geqslant 2$.

Then $\operatorname{ch}_{1}\left(E_{*}\right)=0$.

Proof. In case (a), MacRae [6, Proposition 5.2] shows that $G\left(E_{*}\right)$ is defined by a principal ideal of $R$. Hence, if $G\left(E_{*}\right)$ is a proper ideal, the codimension of the support of $M$ is at most one. Thus $G\left(E_{*}\right)$ is trivial, so, by Theorem $2, \operatorname{ch}_{1}\left(E_{*}\right)=0$.

In case (b), $G\left(E_{*}\right)$ is given locally by a quotient $x / y$, for $x$ and $y$ in $R$, where $R$ is an integrally closed domain, and $\operatorname{Spec} R$ is part of an affine cover of $Y$. Since $R$ is integrally closed and $x / y$ is not a unit in $R$, then the support of $x / y$ has codimension at most one, and the conclusion follows as in part (a).

To prove (c), let $f: Y^{\prime} \rightarrow Y$ be a map of schemes, where, as before, we can assume that $Y^{\prime}$ is an integral scheme of dimension $n$ and we wish to find $\operatorname{ch}_{1}\left(E_{*}\right)\left[Y^{\prime}\right]$. Since $Y^{\prime}$ is integral, it must map into a component of $Y$, and, replacing $Y$ by this component, we can assume that $Y$ is integral as well. Let $\tilde{Y}$ be the normalization of $Y$, and consider the diagram

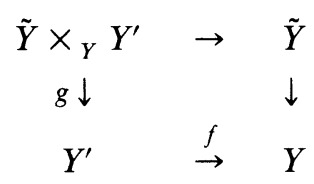

Since $\tilde{Y} \times{ }_{Y} Y^{\prime} \rightarrow Y^{\prime}$ is finite and surjective, there is an integral subscheme $\tilde{Y}^{\prime}$ of $\tilde{Y} \times_{Y} Y^{\prime}$ of dimension $n$ such that $g_{*}\left(\left[\tilde{Y}^{\prime}\right]\right)=m\left[Y^{\prime}\right]$ for some positive integer $m$. Then

$$
\begin{aligned}
m\left(\operatorname{ch}_{1}\left(E_{*}\right)\left[Y^{\prime}\right]\right) & =\operatorname{ch}_{1}\left(E_{*}\right)\left(g_{*}\left(\left[\tilde{Y}^{\prime}\right]\right)\right)=g_{*} \operatorname{ch}_{1}\left(E_{*}\right)\left(\left[\tilde{Y}^{\prime}\right]\right) \\
& =0 \text { by part (b). }
\end{aligned}
$$

Since we are using the Chow groups with rational coefficients, this implies that $\operatorname{ch}_{1}\left(E_{*}\right)=0$, as was to be proven.

\section{REFERENCES}

1. M. Borelli, Some results on ampleness and divisorial schemes, Pacific J. Math. 23 (1967), 217-227.

2. S. Dutta, M. Hochster and J. E. McLaughlin, Modules of finite projective dimension with negative intersection multiplicities, Invent. Math. 79 (1985), 253-291. 
3. H.-B. Foxby, The MacRae invariant, Commutative Algebra (Durham, 1981), London Math. Soc. Lecture Note Series, no. 72, 1982, pp. 121-128.

4. W. Fulton, Intersection theory, Springer-Verlag, Berlin and New York, 1984.

5. A Grothendieck and J. Dieudonne, Éléments de géométrie algébrique, Inst. Hautes Études Sci. Publ. Math. No. 4 (1960).

6. R. E. MacRae, On an application of the Fitting invariants, J. Algebra 2(1965), 153-169.

7. P. Roberts, Local Chern characters and intersection multiplicites, Proc. AMS Conf. Algebraic Geometry, (Bowdoin, Maine, 1985) (to appear).

8. B. Iversen, On determinants of complexes, Aarhus Univ. Preprint Series no. 4, 1979/80.

Department of Mathematics, University of Utah, Salt Lake City, Utah 84112 